\title{
Discrete Modified Projection Methods for Urysohn Integral Equations with Green's Function Type Kernels
}

\section{Rekha P. Kulkarni and Gobinda Rakshit}

\section{Indian Institute of Technology Bombay}

Department of Mathematics, I.I.T. Bombay, Powai, Mumbai 400076, India

E-mail(corresp.): rpk@math.iitb.ac.in

E-mail: gobindarakshit@math.iitb.ac.in

Received August 27, 2019; revised April 25, 2020; accepted April 25, 2020

\begin{abstract}
In the present paper we consider discrete versions of the modified projection methods for solving a Urysohn integral equation with a kernel of the type of Green's function. For $r \geq 0$, a space of piecewise polynomials of degree $\leq r$ with respect to an uniform partition is chosen to be the approximating space. We define a discrete orthogonal projection onto this space and replace the Urysohn integral operator by a Nyström approximation. The order of convergence which we obtain for the discrete version indicates the choice of numerical quadrature which preserves the orders of convergence in the continuous modified projection methods. Numerical results are given for a specific example.
\end{abstract}

Keywords: Urysohn integral operator, orthogonal projection, Nyström approximation, Green's kernel.

AMS Subject Classification: 45G10; 65J15; 65R20.

\section{Introduction}

Let $\mathcal{X}=L^{\infty}[0,1]$ and consider the following nonlinear Urysohn integral equation

$$
x(s)-\int_{0}^{1} \kappa(s, t, x(t)) d t=f(s), \quad s \in[0,1], x \in \mathcal{X},
$$

where $f \in \mathcal{X}$ and the kernel $\kappa(s, t, u)$ is a continuous Green's function type kernel. We write the above equation as

$$
x-\mathcal{K}(x)=f
$$

Copyright (c) 2020 The Author(s). Published by VGTU Press

This is an Open Access article distributed under the terms of the Creative Commons Attribution License (http://creativecommons.org/licenses/by/4.0/), which permits unrestricted use, distribution, and reproduction in any medium, provided the original author and source are credited. 
and assume that it has a unique solution $\varphi$. We are interested in approximate solutions of the above equation.

For $r \geq 0$, let $\mathcal{X}_{n}$ be a space of piecewise polynomials of degree $\leq r$ with respect to a uniform partition of $[0,1]$ with $n$ subintervals each of length $h=\frac{1}{n}$. Let $\pi_{n}$ be the restriction to $L^{\infty}[0,1]$ of the orthogonal projection from $L^{2}[0,1]$ to $\mathcal{X}_{n}$. Then in the classical Galerkin method, (1.2) is approximated by

$$
\varphi_{n}^{G}-\pi_{n} \mathcal{K}\left(\varphi_{n}^{G}\right)=\pi_{n} f
$$

In Krasnoselskii [8], Krasnoselskii et al. [9] and Krasnoselskii-Zabreiko [10], the above projection method has been studied. The iterated Galerkin solution is defined by

$$
\varphi_{n}^{S}=\mathcal{K}\left(\varphi_{n}^{G}\right)+f
$$

The following orders of convergence are proved in Atkinson-Potra [3]:

If $r=0$, then

$$
\left\|\varphi_{n}^{G}-\varphi\right\|_{\infty}=O(h), \quad\left\|\varphi_{n}^{S}-\varphi\right\|_{\infty}=O\left(h^{2}\right)
$$

whereas if $r \geq 1$, then

$$
\left\|\varphi_{n}^{G}-\varphi\right\|_{\infty}=O\left(h^{r+1}\right), \quad\left\|\varphi_{n}^{S}-\varphi\right\|_{\infty}=O\left(h^{r+3}\right) .
$$

In Grammont-Kulkarni [6], the following modified projection method is proposed:

$$
\varphi_{n}^{M}-\mathcal{K}_{n}^{M}\left(\varphi_{n}^{M}\right)=f
$$

where

$$
\mathcal{K}_{n}^{M}(x)=\pi_{n} \mathcal{K}(x)+\mathcal{K}\left(\pi_{n} x\right)-\pi_{n} \mathcal{K}\left(\pi_{n} x\right) .
$$

The iterated modified projection solution is defined as

$$
\tilde{\varphi}_{n}^{M}=\mathcal{K}\left(\varphi_{n}^{M}\right)+f
$$

The following orders of convergence are proved in Grammont et al [7]:

If $r=0$, then

$$
\left\|\varphi_{n}^{M}-\varphi\right\|_{\infty}=O\left(h^{3}\right), \quad\left\|\tilde{\varphi}_{n}^{M}-\varphi\right\|_{\infty}=O\left(h^{4}\right),
$$

whereas if $r \geq 1$, then

$$
\left\|\varphi_{n}^{M}-\varphi\right\|_{\infty}=O\left(h^{r+3}\right), \quad\left\|\tilde{\varphi}_{n}^{M}-\varphi\right\|_{\infty}=O\left(h^{r+5}\right) .
$$

In practice, it is necessary to replace the integral in the definition of $\mathcal{K}$ by a numerical quadrature formula. Also, the orthogonal projection $\pi_{n}$ needs to be replaced by a discrete orthogonal projection $Q_{n}$. This gives rise to discrete versions of the above methods. It is of interest to choose the quadrature formula appropriately so as to preserve the above orders of convergence. Our aim is to investigate the discrete versions of the modified projection and of the iterated modified projection methods. 
The discrete versions of the Galerkin and the iterated Galerkin methods are considered in Atkinson-Potra [4]. They propose a numerical quadrature formula which takes into consideration the fact that the kernel $\kappa(s, t, u)$ lacks smoothness when $s=t$ and obtain the order of convergence of the discrete iterated Galerkin solution.

We follow a different approach. We choose a uniform partition with $m=$ $n p, p \in \mathbb{N}$, subintervals. A composite quadrature formula associated with this fine partition is then used to replace the integrals in the definition of $\mathcal{K}$ and in the definition of the inner product. Let $\tilde{h}=\frac{1}{m}$. Let $z_{n}^{M}$ and $\tilde{z}_{n}^{M}$ denote respectively the discrete modified projection solution and the discrete iterated modified projection solution. We prove the following orders of convergence:

If $r=0$, then

$$
\left\|z_{n}^{M}-\varphi\right\|_{\infty}=O\left(\max \left\{\tilde{h}^{2}, h^{3}\right\}\right), \quad\left\|\tilde{z}_{n}^{M}-\varphi\right\|_{\infty}=O\left(\max \left\{\tilde{h}^{2}, h^{4}\right\}\right),
$$

whereas if $r \geq 1$, then

$$
\left\|z_{n}^{M}-\varphi\right\|_{\infty}=O\left(\max \left\{\tilde{h}^{2}, h^{r+3}\right\}\right),\left\|\tilde{z}_{n}^{M}-\varphi\right\|_{\infty}=O\left(\max \left\{\tilde{h}^{2}, h^{r+5}\right\}\right) .
$$

Thus, if $r=0$ and $\tilde{h}^{2} \leq h^{4}$, that is, $m \geq n^{2}$, then the orders of convergence in (1.3) are preserved. If $r \geq 1$ and $\tilde{h}^{2} \leq h^{r+5}$, then the orders of convergence in (1.4) are preserved.

Note that the term $\tilde{h}^{2}$ in the above estimates appear because of the discretization. If the kernel is smooth, then it is possible to choose a composite quadrature formula associated with the coarse partition with $n$ subintervals and with a precision $d$. Then the term $\tilde{h}^{2}$ is replaced by $h^{d}$ and an appropriate choice of $d$ will preserve the orders of convergence in (1.3) and (1.4). However, in the case of the kernel of the type of Green's function, the error in the higher order quadrature rules also is only of the order of $h^{2}$. Hence we need to choose a different partition for the quadrature rule which makes the proofs more involved. It is to be noted that even if $m>n$, the size of the system of equations that need to be solved in order to compute $z_{n}^{M}$ remains $n(r+1)$.

Note that in Grammont et al [7], the orders of convergence (1.3) and (1.4) for the (continuous) modified projection and the iterated modified projection are proved. However, the numerical results are given for the discrete versions of the modified projection and of the iterated modified projection methods. In the present paper we fill this gap and justify the numerical results of Grammont et al [7].

The paper has been arranged in the following way. In Section 2, we define a discrete orthogonal projection operator and discrete versions of the modified projection methods. In Section 3 , we consider the case of a piecewise polynomial space of degree $r \geq 1$ and prove (1.6). Section 4, is devoted to the proof of (1.5) in the case of piecewise constant functions. Numerical results for illustrative purpose are given in Section 5 .

\section{Discrete modified projection method}

In this section we describe the Nyström approximation of $\mathcal{K}$ and the discrete orthogonal projection. We then define discrete analogues of the modified pro- 
jection method and its iterated version.

\subsection{Kernel of the type of Green's function}

Let $r \geq 0$ be an integer and assume that the kernel $\kappa$ of the integral operator $\mathcal{K}$ appearing in (1.1) has the following properties.

1. Let $\Psi=[0,1] \times[0,1] \times \mathbb{R}$. The partial derivative $\ell(s, t, u)=\frac{\partial \kappa(s, t, u)}{\partial u}$ is continuous for all $(s, t, u) \in \Psi$.

2. Let $\Psi_{1}=\{(s, t, u): 0 \leq t \leq s \leq 1, u \in \mathbb{R}\}, \quad \Psi_{2}=\{(s, t, u): 0 \leq s \leq t \leq$ $1, u \in \mathbb{R}\}$. There are functions $\ell_{i} \in C^{r+1}\left(\Psi_{i}\right), i=1,2$, with

$$
\ell(s, t, u)= \begin{cases}\ell_{1}(s, t, u), & (s, t, u) \in \Psi_{1}, \\ \ell_{2}(s, t, u), & (s, t, u) \in \Psi_{2}\end{cases}
$$

3. There are functions $\kappa_{i} \in C^{r+1}\left(\Psi_{i}\right), i=1,2$, such that

$$
\kappa(s, t, u)= \begin{cases}\kappa_{1}(s, t, u), & (s, t, u) \in \Psi_{1} \\ \kappa_{2}(s, t, u), & (s, t, u) \in \Psi_{2}\end{cases}
$$

4. $\partial^{2} \kappa / \partial u^{2} \in C(\Psi)$.

Following Atkinson-Potra [3], if the kernel $\kappa$ satisfies the above conditions, then we say that $\kappa$ is of class $\mathcal{G}_{2}(r+1,0)$.

Let $f \in C^{r+1}[0,1]$. Then by the Corollary 3.2 of Atkinson-Potra [3], it follows that $\varphi \in C^{r+1}[0,1]$. If $r=0$, then it is assumed that $f \in C^{2}[0,1]$ so that $\varphi \in C^{2}[0,1]$. We assume that $\mathcal{K}$ is twice Fréchet differentiable and that 1 is not an eigenvalue of $\mathcal{K}^{\prime}(\varphi)$.

\subsection{Nyström approximation}

Let $m \in \mathbb{N}$ and consider the following uniform partition of $[0,1]$ :

$$
0<1 / m<\cdots<(m-1) / m<1 \text {. }
$$

Let $\tilde{h}=\frac{1}{m}$ and $s_{i}=\frac{i}{m}, \quad i=0, \ldots, m$. Consider a basic quadrature rule of the form

$$
\int_{0}^{1} f(t) d t \approx \sum_{q=1}^{\rho} w_{q} f\left(\mu_{q}\right)
$$

where the weights $w_{q}>0$ and the nodes $\mu_{q} \in[0,1]$. It is assumed that the quadrature rule is exact at least for polynomials of degree $\leq 2 r$.

A composite integration rule with respect to the partition (2.1) is then defined as

$$
\int_{0}^{1} f(t) d t=\sum_{i=1}^{m} \int_{s_{i-1}}^{s_{i}} f(t) d t \approx \tilde{h} \sum_{i=1}^{m} \sum_{q=1}^{\rho} w_{q} f\left(\zeta_{q}^{i}\right), \quad \zeta_{q}^{i}=s_{i-1}+\mu_{q} \tilde{h} .
$$


We replace the integral in (1.1) by the numerical quadrature formula (2.2) and define the Nyström operator as

$$
\mathcal{K}_{m}(x)(s)=\tilde{h} \sum_{i=1}^{m} \sum_{q=1}^{\rho} w_{q} \kappa\left(s, \zeta_{q}^{i}, x\left(\zeta_{q}^{i}\right)\right), \quad s \in[0,1] .
$$

Note that $\mathcal{K}_{m}$ is twice Fréchet differentiable and

$$
\mathcal{K}_{m}^{\prime}(x) v(s)=\tilde{h} \sum_{i=1}^{m} \sum_{q=1}^{\rho} w_{q} \frac{\partial \kappa}{\partial u}\left(s, \zeta_{q}^{i}, x\left(\zeta_{q}^{i}\right)\right) v\left(\zeta_{q}^{i}\right), \quad s \in[0,1], v \in \mathcal{X},
$$

and for $v_{1}, v_{2} \in \mathcal{X}$,

$$
\mathcal{K}_{m}^{\prime \prime}(x)\left(v_{1}, v_{2}\right)(s)=\tilde{h} \sum_{i=1}^{m} \sum_{q=1}^{\rho} w_{q} \frac{\partial^{2} \kappa}{\partial u^{2}}\left(s, \zeta_{q}^{i}, x\left(\zeta_{q}^{i}\right)\right) v_{1}\left(\zeta_{q}^{i}\right) v_{2}\left(\zeta_{q}^{i}\right), \quad s \in[0,1] .
$$

For $\delta_{0}>0$, let $\mathcal{B}\left(\varphi, \delta_{0}\right)=\left\{\psi \in \mathcal{X}:\|\varphi-\psi\|_{\infty}<\delta_{0}\right\}$. Define

$$
C_{1}=\max _{\substack{s, t \in[0,1] \\|u| \leq\|\varphi \varphi\|_{\infty}+\delta_{0}}}\left|\frac{\partial \kappa}{\partial u}(s, t, u)\right| \text { and } C_{2}=\max _{\substack{s, t \in[0,1] \\|u| \leq\|\| \|_{\infty}+\delta_{0}}}\left|\frac{\partial^{2} \kappa}{\partial u^{2}}(s, t, u)\right| \text {. }
$$

Then for $x, y \in \mathcal{B}\left(\varphi, \delta_{0}\right)$,

$$
\begin{aligned}
& \left\|\mathcal{K}_{m}^{\prime}(x) v\right\|_{\infty} \leq C_{1}\|v\|_{\infty}, \\
& \left\|\mathcal{K}_{m}^{\prime}(x)-\mathcal{K}_{m}^{\prime}(y)\right\| \leq C_{2}\|x-y\|_{\infty} .
\end{aligned}
$$

If $T: \mathcal{X} \rightarrow \mathcal{X}$ is a bounded linear operator, then $\|T\|=\sup \left\{\|T v\|_{\infty}:\|v\|_{\infty} \leq 1\right\}$ denotes the operator norm.

Since the kernel lacks smoothness along $s=t$, we only have the following order of convergence from Atkinson-Potra [4]: If $x \in C^{2}[0,1]$, then

$$
\left\|\mathcal{K}(x)-\mathcal{K}_{m}(x)\right\|_{\infty}=O\left(\tilde{h}^{2}\right) .
$$

In the Nyström method, (1.2) is approximated by

$$
x_{m}-\mathcal{K}_{m}\left(x_{m}\right)=f .
$$

For all $m$ big enough, the above equation has a unique solution $\varphi_{m}$ in $\mathcal{B}\left(\varphi, \delta_{0}\right)$ and

$$
\left\|\varphi-\varphi_{m}\right\|_{\infty} \leq C_{3}\left\|\mathcal{K}(\varphi)-\mathcal{K}_{m}(\varphi)\right\|_{\infty}=O\left(\tilde{h}^{2}\right)
$$

See Atkinson [1]. We quote the following result from Krasnoselskii et al [9] for future reference:

If $v_{1}, v_{2} \in \mathcal{B}\left(\varphi, \delta_{0}\right), s \in[0,1]$, then by the generalized Taylor's theorem,

$$
\mathcal{K}_{m}\left(v_{2}\right)(s)-\mathcal{K}_{m}\left(v_{1}\right)(s)-\mathcal{K}_{m}^{\prime}\left(v_{1}\right)\left(v_{2}-v_{1}\right)(s)=R\left(v_{2}-v_{1}\right)(s),
$$

where

$$
R\left(v_{2}-v_{1}\right)(s)=\int_{0}^{1}(1-\theta) \mathcal{K}_{m}^{\prime \prime}\left(v_{1}+\theta\left(v_{2}-v_{1}\right)\right)\left(v_{2}-v_{1}\right)^{2}(s) d \theta .
$$

It then follows that

$$
\left\|R\left(v_{2}-v_{1}\right)\right\|_{\infty} \leq C_{2}\left\|v_{2}-v_{1}\right\|_{\infty}^{2}
$$




\subsection{Discrete orthogonal projection}

Let $n \in \mathbb{N}$ and consider the following uniform partition of $[0,1]$ :

$$
\Delta: 0<1 / n<\cdots<(n-1) / n<1 \text {. }
$$

Define

$$
t_{j}=j / n, \quad \Delta_{j}=\left[t_{j-1}, t_{j}\right] \text { and } h=t_{j}-t_{j-1}=1 / n, \quad j=1, \ldots, n .
$$

For $r \geq 0$, let $\mathcal{X}_{n}$ denote the space of piecewise polynomials of degree $\leq r$ with respect to the partition of $(2.9)$ of $[0,1]$. Assume that the values at $t_{j}-, j=$ $1, \ldots, n$, are defined by continuity. Then the dimension of $\mathcal{X}_{n}$ is $n(r+1)$.

For $\eta=0,1, \ldots, r$, let $L_{\eta}$ denote the Legendre polynomial of degree $\eta$ on $[-1,1]$. For $j=1, \ldots, n$, and for $\eta=0,1, \ldots, r$, define

$$
\varphi_{j, \eta}(t)=\left\{\begin{array}{l}
\sqrt{\frac{2}{h}} L_{\eta}\left(\frac{2 t-t_{j}-t_{j-1}}{h}\right), \quad t \in\left(t_{j-1}, t_{j}\right], \quad \varphi_{1, \eta}\left(t_{0}\right)=\sqrt{\frac{2}{h}} L_{\eta}(-1) . \\
0, \quad \text { otherwise },
\end{array}\right.
$$

Note that $\left\|\varphi_{j, \eta}\right\|_{\infty}=\max _{t \in\left[t_{j-1}, t_{j}\right]}\left|\varphi_{j, \eta}(t)\right|=\sqrt{\frac{2}{h}}\left\|L_{\eta}\right\|_{\infty}$.

Assume that $m=p n$ for some $p \in \mathbb{N}$. For $f, g \in C\left(\Delta_{j}\right)$, define

$$
\langle f, g\rangle_{\Delta_{j}}=\tilde{h} \sum_{\nu=1}^{p} \sum_{q=1}^{\rho} w_{q} f\left(\zeta_{q}^{(j-1) p+\nu}\right) g\left(\zeta_{q}^{(j-1) p+\nu}\right)
$$

where $\zeta_{q}^{(j-1) p+\nu}$ are defined in (2.2). Note that $\langle f, g\rangle_{\Delta_{j}}=\langle g, f\rangle_{\Delta_{j}}$. Define $\|f\|_{\Delta_{j}, \infty}=\max _{t \in\left[t_{j-1}, t_{j}\right]}|f(t)|$. Then

$$
\left|\langle f, g\rangle_{\Delta_{j}}\right| \leq\|f\|_{\Delta_{j}, \infty}\|g\|_{\Delta_{j}, \infty} h .
$$

Since the quadrature rule is exact for polynomials of degree $\leq 2 r$, we have

$$
\delta_{\eta, \eta^{\prime}}=\left\langle\varphi_{j, \eta}, \varphi_{j, \eta^{\prime}}\right\rangle=\int_{0}^{1} \varphi_{j, \eta}(t) \varphi_{j, \eta^{\prime}}(t) d t=\left\langle\varphi_{j, \eta}, \varphi_{j, \eta^{\prime}}\right\rangle_{\Delta_{j}} .
$$

Thus, $\left\{\varphi_{j, \eta}, j=1, \ldots, n, \eta=0, \ldots, r\right\}$ forms an orthonormal basis for $\mathcal{X}_{n}$. Let $\mathcal{P}_{r, \Delta_{j}}$ denote the space of polynomials of degree $\leq r$ on $\Delta_{j}$. Define the discrete orthogonal projection $Q_{n, j}: C\left(\Delta_{j}\right) \rightarrow \mathcal{P}_{r, \Delta_{j}}$ as follows:

$$
Q_{n, j} x=\sum_{\eta=0}^{r}\left\langle x, \varphi_{j, \eta}\right\rangle_{\Delta_{j}} \varphi_{j, \eta}
$$

Then $\left\langle Q_{n, j} x, y\right\rangle_{\Delta_{j}}=\left\langle x, Q_{n, j} y\right\rangle_{\Delta_{j}}, Q_{n, j}^{2}=Q_{n, j}$ and $Q_{n, j} Q_{n, i}=0$ for $i \neq j$. Also,

$$
\left\|Q_{n, j} x\right\|_{\Delta_{j}, \infty} \leq \sum_{\eta=0}^{r}\left|\left\langle x, \varphi_{j, \eta}\right\rangle_{\Delta_{j}}\right|\left\|\varphi_{j, \eta}\right\|_{\Delta_{j}, \infty} \leq\left(2 \sum_{\eta=0}^{r}\left\|L_{\eta}\right\|_{\infty}^{2}\right)\|x\|_{\infty} .
$$


A discrete orthogonal projection $Q_{n}: C[0,1] \rightarrow \mathcal{X}_{n}$ is defined as follows:

$$
Q_{n} x=\sum_{j=1}^{n} Q_{n, j} x
$$

Using the Hahn-Banach extension theorem, as in Atkinson et al [2], $Q_{n}$ can be extended to $L^{\infty}[0,1]$. Then

$$
Q_{n}^{2}=Q_{n} \quad \text { and } \quad\left\|Q_{n}\right\| \leq 2 \sum_{\eta=0}^{r}\left\|L_{\eta}\right\|_{\infty}^{2}=C_{4} .
$$

The following estimate is standard: if $x \in C^{r+1}\left(\Delta_{j}\right)$, then we have,

$$
\left\|\left(I-Q_{n, j}\right) x\right\|_{\Delta_{j}, \infty} \leq C_{5}\left\|x^{(r+1)}\right\|_{\Delta_{j}, \infty} h^{r+1} .
$$

Thus, if $x \in C^{r+1}[0,1]$, then

$$
\left\|\left(I-Q_{n}\right) x\right\|_{\infty}=O\left(h^{r+1}\right) .
$$

\subsection{Discrete projection methods}

We define below the discrete versions of various projection methods given in Section 1 by replacing the integral operator $\mathcal{K}$ by the Nyström operator $\mathcal{K}_{m}$ and the orthogonal projection $\pi_{n}$ by the discrete orthogonal projection $Q_{n}$.

Discrete Galerkin Method: $z_{n}^{G}-Q_{n} \mathcal{K}_{m}\left(z_{n}^{G}\right)=Q_{n} f$.

Discrete Iterated Galerkin Method: $z_{n}^{S}-\mathcal{K}_{m}\left(Q_{n} z_{n}^{S}\right)=f$.

The discrete modified projection operator is defined as

$$
\tilde{\mathcal{K}}_{n}^{M}(x)=Q_{n} \mathcal{K}_{m}(x)+\mathcal{K}_{m}\left(Q_{n} x\right)-Q_{n} \mathcal{K}_{m}\left(Q_{n} x\right) .
$$

Discrete Modified Projection method:

$$
z_{n}^{M}-\tilde{\mathcal{K}}_{n}^{M}\left(z_{n}^{M}\right)=f
$$

Discrete Iterated Modified Projection method:

$$
\tilde{z}_{n}^{M}=\mathcal{K}_{m}\left(z_{n}^{M}\right)+f .
$$

\section{Piecewise polynomial approximation: $r \geq 1$}

In this section we consider the case $r \geq 1$ and obtain orders of convergence in the discrete modified projection method and its iterated version.

\subsection{Preliminary results}

Let $\ell_{*}(s, t)=\ell(s, t, \varphi(t)), \quad 0 \leq s, t \leq 1$. Then

$$
\ell_{*}(s, t)= \begin{cases}\ell_{1, *}(s, t)=\ell_{1}(s, t, \varphi(t)), & 0 \leq t \leq s \leq 1 \\ \ell_{2, *}(s, t)=\ell_{2}(s, t, \varphi(t)), & 0 \leq s \leq t \leq 1\end{cases}
$$


Since $\varphi \in C^{r+1}[0,1]$, it follows that

$$
\ell_{1, *} \in C^{r+1}(\{0 \leq t \leq s \leq 1\}) \text { and } \ell_{2, *} \in C^{r+1}(\{0 \leq s \leq t \leq 1\}) .
$$

We introduce the following notation.

For a fixed $s \in[0,1]$, define $\ell_{*, s}(t)=\ell_{*}(s, t), t \in[0,1]$. Note that

$$
\mathcal{K}_{m}^{\prime}(\varphi) v(s)=\tilde{h} \sum_{j=1}^{n} \sum_{\nu=1}^{p} \sum_{q=1}^{\rho} w_{q} \ell_{*}\left(s, \zeta_{q}^{(j-1) p+\nu}\right) v\left(\zeta_{q}^{(j-1) p+\nu}\right)=\sum_{j=1}^{n}\left\langle\ell_{*, s}, v\right\rangle_{\Delta_{j}} .
$$

Let

$$
C_{6}=\max _{1 \leq j \leq r+1}\left\{\max _{\substack{0 \leq t \leq s \leq 1 \\|u| \leq\|\varphi\|_{\infty}}}\left|D^{(0, j, 0)} \ell_{1}(s, t, u)\right|, \max _{\substack{0 \leq s \leq t \leq 1 \\|u| \leq\|\| \varphi \|_{\infty}}}\left|D^{(0, j, 0)} \ell_{2}(s, t, u)\right|\right\} .
$$

The following proposition is crucial. It will be used several times in what follows.

Proposition 1. If $v \in C^{r+1}[0,1]$, then

$$
\left\|\mathcal{K}_{m}^{\prime}(\varphi)\left(I-Q_{n}\right) v\right\|_{\infty} \leq\left(C_{5}\right)^{2} C_{6}\left\|v^{(r+1)}\right\|_{\infty} h^{r+3}
$$

Proof. For $s \in[0,1]$,

$$
\begin{aligned}
\mathcal{K}_{m}^{\prime}(\varphi)\left(I-Q_{n}\right) v(s) & =\sum_{j=1}^{n}\left\langle\ell_{*, s},\left(I-Q_{n, j}\right) v\right\rangle_{\Delta_{j}} \\
& =\sum_{j=1}^{n}\left\langle\left(I-Q_{n, j}\right) \ell_{*, s},\left(I-Q_{n, j}\right) v\right\rangle_{\Delta_{j}} .
\end{aligned}
$$

Case 1: $s=t_{i}$ for some $i \in\{0,1, \ldots, n\}$. Then $\ell_{*, s} \in C^{r+1}\left(\Delta_{j}\right)$ for $j=1, \ldots, n$. Since $v \in C^{r+1}[0,1]$, it follows from $(2.14)$,

$$
\max _{0 \leq i \leq n}\left|\mathcal{K}_{m}^{\prime}(\varphi)\left(I-Q_{n}\right) v\left(t_{i}\right)\right| \leq\left(C_{5}\right)^{2} C_{6}\left\|v^{(r+1)}\right\|_{\infty} h^{2 r+2} .
$$

Case 2: $s \in\left(t_{i-1}, t_{i}\right)$ for some $i \in\{1,2, \ldots, n\}$. We write

$$
\begin{aligned}
\mathcal{K}_{m}^{\prime}(\varphi)\left(I-Q_{n}\right) v(s)= & \sum_{\substack{j=1 \\
j \neq i}}^{n}\left\langle\left(I-Q_{n, j}\right) \ell_{*, s},\left(I-Q_{n, j}\right) v\right\rangle_{\Delta_{j}} \\
& +\left\langle\left(I-Q_{n, i}\right) \ell_{*, s},\left(I-Q_{n, i}\right) v\right\rangle_{\Delta_{i}} .
\end{aligned}
$$

For $j \neq i, \ell_{*, s} \in C^{r+1}\left(\Delta_{j}\right)$ and $v \in C^{r+1}\left(\Delta_{j}\right)$. Hence

$$
\left|\sum_{\substack{j=1 \\ j \neq i}}^{n}\left\langle\left(I-Q_{n, j}\right) \ell_{*, s},\left(I-Q_{n, j}\right) v\right\rangle_{\Delta_{j}}\right| \leq\left(C_{5}\right)^{2} C_{6}\left\|v^{(r+1)}\right\|_{\infty}(n-1) h^{2 r+3} .
$$


We now consider the case $j=i$. Note that $\ell_{*, s}$ is only continuous on $\left[t_{i-1}, t_{i}\right]$. Define a constant function: $g_{i}(t)=\ell_{*, s}(s), t \in\left[t_{i-1}, t_{i}\right]$. Note that

$$
\left\langle\left(I-Q_{n, i}\right) \ell_{*, s},\left(I-Q_{n, i}\right) v\right\rangle_{\Delta_{i}}=\left\langle\ell_{*, s}-g_{i},\left(I-Q_{n, i}\right) v\right\rangle_{\Delta_{i}}
$$

For $t \in\left[t_{i-1}, t_{i}\right]$,

$$
\ell_{*, s}(t)-g_{i}(t)= \begin{cases}D^{(0,1)} \ell_{1, *}\left(s, \theta_{t}\right)(t-s), & \theta_{t} \in(t, s) \\ D^{(0,1)} \ell_{2, *}\left(s, \eta_{t}\right)(t-s), & \eta_{t} \in(s, t) .\end{cases}
$$

Thus,

$$
\left|\left\langle\left(I-Q_{n, i}\right) \ell_{*, s},\left(I-Q_{n, i}\right) v\right\rangle_{\Delta_{i}}\right| \leq C_{5} C_{6}\left\|v^{(r+1)}\right\|_{\infty} h^{r+3} .
$$

Without loss of generality, let $C_{5} \geq 1$. From (3.3), (3.4) and (3.5) we obtain,

$$
\left|\mathcal{K}_{m}^{\prime}(\varphi)\left(I-Q_{n}\right) v(s)\right| \leq\left(C_{5}\right)^{2} C_{6}\left\|v^{(r+1)}\right\|_{\infty} h^{r+3}
$$

Combining the above estimate with (3.2) we obtain the required result.

Proposition 2. If $v \in C^{r+1}[0,1]$, then

$$
\left\|\mathcal{K}_{m}^{\prime}(\varphi)\left(I-Q_{n}\right) \mathcal{K}_{m}^{\prime}(\varphi)\left(I-Q_{n}\right) v\right\|_{\infty}=O\left(h^{r+5}\right) .
$$

Also,

$$
\left\|\mathcal{K}_{m}^{\prime}(\varphi)\left(I-Q_{n}\right) \mathcal{K}_{m}^{\prime}(\varphi)\right\|=O\left(h^{2}\right)
$$

Proof. The proof of (3.6) is similar to that of (3.1). For $s \in[0,1]$, we write

$$
\mathcal{K}_{m}^{\prime}(\varphi)\left(I-Q_{n}\right) \mathcal{K}_{m}^{\prime}(\varphi)\left(I-Q_{n}\right) v(s)=\sum_{j=1}^{n}\left\langle\left(I-Q_{n, j}\right) \ell_{*, s}, \mathcal{K}_{m}^{\prime}(\varphi)\left(I-Q_{n}\right) v\right\rangle_{\Delta_{j}} .
$$

If $s=t_{i}$, for some $i$, then using (2.14) and (3.1) we obtain

$$
\left|\mathcal{K}_{m}^{\prime}(\varphi)\left(I-Q_{n}\right) \mathcal{K}_{m}^{\prime}(\varphi)\left(I-Q_{n}\right) v\left(t_{i}\right)\right| \leq\left(C_{5}\right)^{3}\left(C_{6}\right)^{2}\left\|v^{(r+1)}\right\|_{\infty} h^{2 r+4} .
$$

If $s \in\left(t_{i-1}, t_{i}\right)$, then we write

$$
\begin{gathered}
\mathcal{K}_{m}^{\prime}(\varphi)\left(I-Q_{n}\right) \mathcal{K}_{m}^{\prime}(\varphi)\left(I-Q_{n}\right) v(s)=\sum_{\substack{j=1 \\
j \neq i}}^{n}\left\langle\left(I-Q_{n, j}\right) \ell_{*, s}, \mathcal{K}_{m}^{\prime}(\varphi)\left(I-Q_{n}\right) v\right\rangle_{\Delta_{j}} \\
+\left\langle\ell_{*, s}-g_{i}, \mathcal{K}_{m}^{\prime}(\varphi)\left(I-Q_{n}\right) v\right\rangle_{\Delta_{i}} .
\end{gathered}
$$

Proceeding as in the proof of Proposition 1, we obtain

$$
\left|\mathcal{K}_{m}^{\prime}(\varphi)\left(I-Q_{n}\right) \mathcal{K}_{m}^{\prime}(\varphi)\left(I-Q_{n}\right) v(s)\right| \leq\left(C_{5}\right)^{3}\left(C_{6}\right)^{2}\left\|v^{(r+1)}\right\|_{\infty} h^{r+5} .
$$

The estimate (3.6) follows from the above two estimates. In order to prove (3.7), consider $v \in C[0,1]$. Let $s=t_{i}$ for some $i$. Then

$$
\left|\mathcal{K}_{m}^{\prime}(\varphi)\left(I-Q_{n}\right) v(s)\right| \leq C_{5} C_{6}\|v\|_{\infty} h^{r+1} .
$$


Now let $s \in\left(t_{i-1}, t_{i}\right)$. We write

$$
\mathcal{K}_{m}^{\prime}(\varphi)\left(I-Q_{n}\right) v(s)=\sum_{\substack{j=1 \\ j \neq i}}^{n}\left\langle\left(I-Q_{n, j}\right) \ell_{*, s}, v\right\rangle_{\Delta_{j}}+\left\langle\left(I-Q_{n, i}\right)\left(\ell_{*, s}-g_{i}\right), v\right\rangle_{\Delta_{i}}
$$

and obtain

$$
\left|\mathcal{K}_{m}^{\prime}(\varphi)\left(I-Q_{n}\right) v(s)\right| \leq\left(1+C_{4}+C_{5}\right) C_{6}\|v\|_{\infty} h^{2} .
$$

Combining (3.8) and the above estimate, we obtain

$$
\left\|\mathcal{K}_{m}^{\prime}(\varphi)\left(I-Q_{n}\right) v\right\|_{\infty} \leq\left(1+C_{4}+C_{5}\right) C_{6}\|v\|_{\infty} h^{2} .
$$

Since from $(2.3),\left\|\mathcal{K}_{m}^{\prime}(\varphi) v\right\|_{\infty} \leq C_{1}\|v\|_{\infty}$, we obtain

$$
\left\|\mathcal{K}_{m}^{\prime}(\varphi)\left(I-Q_{n}\right) \mathcal{K}_{m}^{\prime}(\varphi) v\right\|_{\infty} \leq C_{1}\left(1+C_{4}+C_{5}\right) C_{6}\|v\|_{\infty} h^{2}
$$

and the required result follows taking the supremum over unit ball in $C[0,1]$.

\subsection{Error in the discrete modified projection method}

As in Grammont [5], it can be shown that there is a $\delta_{0}>0$ such that $(2.16)$ has a unique solution $z_{n}^{M}$ in $\mathcal{B}\left(\varphi, \delta_{0}\right)$ and that

$$
\begin{aligned}
& \left\|z_{n}^{M}-\varphi\right\|_{\infty} \\
& \leq 6\left\|\left(I-\mathcal{K}^{\prime}(\varphi)\right)^{-1}\right\|\left(\left\|\mathcal{K}(\varphi)-\mathcal{K}_{m}(\varphi)\right\|_{\infty}+\left\|\mathcal{K}_{m}(\varphi)-\tilde{\mathcal{K}}_{n}^{M}(\varphi)\right\|_{\infty}\right) .
\end{aligned}
$$

In the following theorem, we obtain the order of convergence of the discrete modified projection solution.

Theorem 1. Let $r \geq 1, \kappa$ be of class $\mathcal{G}_{2}(r+1,0)$ and $f \in C^{r+1}[0,1]$. Let $\varphi$ be the unique solution of (1.2) and assume that 1 is not an eigenvalue of $\mathcal{K}^{\prime}(\varphi)$. Let $\mathcal{X}_{n}$ be the space of piecewise polynomials of degree $\leq r$ with respect to the partition (2.9) and $Q_{n}$ be the discrete orthogonal projection defined by (2.12). Let $z_{n}^{M}$ be the discrete modified projection solution in $\mathcal{B}\left(\varphi, \delta_{0}\right)$. Then

$$
\left\|z_{n}^{M}-\varphi\right\|_{\infty}=O\left(\max \left\{\tilde{h}^{2}, h^{r+3}\right\}\right) .
$$

Proof. From (2.5),

$$
\left\|\mathcal{K}(\varphi)-\mathcal{K}_{m}(\varphi)\right\|_{\infty}=O\left(\tilde{h}^{2}\right)
$$

Since $\varphi \in C^{r+1}[0,1]$, it follows from $(2.15)$ that $\left\|Q_{n} \varphi-\varphi\right\|_{\infty}=O\left(h^{r+1}\right)$. Note that

$$
\begin{aligned}
\left\|\mathcal{K}_{m}(\varphi)-\tilde{\mathcal{K}}_{n}^{M}(\varphi)\right\|_{\infty} \leq & \left\|\left(I-Q_{n}\right)\left(\mathcal{K}_{m}\left(Q_{n} \varphi\right)-\mathcal{K}_{m}(\varphi)-\mathcal{K}_{m}^{\prime}(\varphi)\left(Q_{n} \varphi-\varphi\right)\right)\right\|_{\infty} \\
& +\left\|\left(I-Q_{n}\right) \mathcal{K}_{m}^{\prime}(\varphi)\left(Q_{n} \varphi-\varphi\right)\right\|_{\infty} .
\end{aligned}
$$


From (2.7), (2.8) and (2.15),

$$
\left\|\mathcal{K}_{m}\left(Q_{n} \varphi\right)-\mathcal{K}_{m}(\varphi)-\mathcal{K}_{m}^{\prime}(\varphi)\left(Q_{n} \varphi-\varphi\right)\right\|_{\infty} \leq C_{2}\left\|Q_{n} \varphi-\varphi\right\|_{\infty}^{2}=O\left(h^{2 r+2}\right) .
$$

By (2.13) and Proposition 1,

$$
\left\|\left(I-Q_{n}\right) \mathcal{K}_{m}^{\prime}(\varphi)\left(Q_{n} \varphi-\varphi\right)\right\|_{\infty} \leq\left(1+C_{4}\right)\left\|\mathcal{K}_{m}^{\prime}(\varphi)\left(Q_{n} \varphi-\varphi\right)\right\|_{\infty}=O\left(h^{r+3}\right) .
$$

Since $r \geq 1$, it follows that $\left\|\mathcal{K}_{m}(\varphi)-\tilde{\mathcal{K}}_{n}^{M}(\varphi)\right\|_{\infty}=O\left(h^{r+3}\right)$.

The required result follows from (3.10), (3.12) and the above estimate.

Remark 1. It can be shown that

$$
\left\|z_{n}^{G}-\varphi\right\|_{\infty}=O\left(\max \left\{\tilde{h}^{2}, h^{r+1}\right\}\right),\left\|z_{n}^{S}-\varphi\right\|_{\infty}=O\left(\max \left\{\tilde{h}^{2}, h^{r+3}\right\}\right) .
$$

\subsection{Error in the discrete iterated modified projection method}

Note that $\tilde{z}_{n}^{M}-\varphi_{m}=\mathcal{K}_{m}\left(z_{n}^{M}\right)-\mathcal{K}_{m}\left(\varphi_{m}\right)$. From (2.7) and (2.8),

$$
\mathcal{K}_{m}\left(z_{n}^{M}\right)-\mathcal{K}_{m}\left(\varphi_{m}\right)=\mathcal{K}_{m}^{\prime}\left(\varphi_{m}\right)\left(z_{n}^{M}-\varphi_{m}\right)+O\left(\left\|z_{n}^{M}-\varphi_{m}\right\|_{\infty}^{2}\right) .
$$

From (2.6) and Theorem 1, we obtain

$$
\left\|z_{n}^{M}-\varphi_{m}\right\|_{\infty} \leq\left\|z_{n}^{M}-\varphi\right\|_{\infty}+\left\|\varphi-\varphi_{m}\right\|_{\infty}=O\left(\max \left\{\tilde{h}^{2}, h^{r+3}\right\}\right) .
$$

Thus,

$$
\tilde{z}_{n}^{M}-\varphi_{m}=\mathcal{K}_{m}^{\prime}\left(\varphi_{m}\right)\left(z_{n}^{M}-\varphi_{m}\right)+O\left(\max \left\{\tilde{h}^{2}, h^{r+3}\right\}^{2}\right) .
$$

Let

$$
L_{m}=\left[I-\mathcal{K}_{m}^{\prime}\left(\varphi_{m}\right)\right]^{-1} \mathcal{K}_{m}^{\prime}\left(\varphi_{m}\right) .
$$

We quote the following result from Kulkarni-Rakshit [11]:

$$
\begin{aligned}
& \mathcal{K}_{m}^{\prime}\left(\varphi_{m}\right)\left(z_{n}^{M}-\varphi_{m}\right)=-L_{m}\left\{\mathcal{K}_{m}\left(\varphi_{m}\right)-\tilde{\mathcal{K}}_{n}^{M}\left(\varphi_{m}\right)\right\} \\
& +L_{m}\left\{\tilde{\mathcal{K}}_{n}^{M}\left(z_{n}^{M}\right)-\tilde{\mathcal{K}}_{n}^{M}\left(\varphi_{m}\right)-\left(\tilde{\mathcal{K}}_{n}^{M}\right)^{\prime}\left(\varphi_{m}\right)\left(z_{n}^{M}-\varphi_{m}\right)\right\} \\
& +L_{m}\left\{\left(\left(\tilde{\mathcal{K}}_{n}^{M}\right)^{\prime}\left(\varphi_{m}\right)-\mathcal{K}_{m}^{\prime}\left(\varphi_{m}\right)\right)\left(z_{n}^{M}-\varphi_{m}\right)\right\} .
\end{aligned}
$$

We obtain below orders of convergence for the three terms in (3.15).

Proposition 3. Let $\varphi_{m}$ be the Nyström solution. Then

$$
\left\|\mathcal{K}_{m}^{\prime}\left(\varphi_{m}\right)\left(\mathcal{K}_{m}\left(\varphi_{m}\right)-\tilde{\mathcal{K}}_{n}^{M}\left(\varphi_{m}\right)\right)\right\|_{\infty}=O\left(h^{4} \max \left\{\tilde{h}^{2}, h^{r+1}\right\}\right) .
$$

Proof. Let $v \in C[0,1]$. Then from (2.4), (2.6) and (3.9),

$$
\begin{aligned}
\| \mathcal{K}_{m}^{\prime}\left(\varphi_{m}\right) & \left(I-Q_{n}\right) v \|_{\infty} \\
& \leq\left\|\left[\mathcal{K}_{m}^{\prime}\left(\varphi_{m}\right)-\mathcal{K}_{m}^{\prime}(\varphi)\right]\left(I-Q_{n}\right) v\right\|_{\infty}+\left\|\mathcal{K}_{m}^{\prime}(\varphi)\left(I-Q_{n}\right) v\right\|_{\infty} \\
& \leq C_{2}\left(1+C_{4}\right)\|v\|_{\infty}\left\|\varphi_{m}-\varphi\right\|_{\infty}+\left(1+C_{4}+C_{5}\right) C_{6}\|v\|_{\infty} h^{2} \\
& \leq C_{7}\|v\|_{\infty} h^{2} .
\end{aligned}
$$


Note that

$$
\begin{aligned}
& \mathcal{K}_{m}\left(\varphi_{m}\right)-\tilde{\mathcal{K}}_{n}^{M}\left(\varphi_{m}\right)=-\left(I-Q_{n}\right)\left(\mathcal{K}_{m}\left(Q_{n} \varphi_{m}\right)-\mathcal{K}_{m}\left(\varphi_{m}\right)\right. \\
& \left.\quad-\mathcal{K}_{m}^{\prime}\left(\varphi_{m}\right)\left(Q_{n} \varphi_{m}-\varphi_{m}\right)\right)-\left(I-Q_{n}\right) \mathcal{K}_{m}^{\prime}\left(\varphi_{m}\right)\left(Q_{n} \varphi_{m}-\varphi_{m}\right) .
\end{aligned}
$$

Let

$$
y_{n}=\mathcal{K}_{m}\left(Q_{n} \varphi_{m}\right)-\mathcal{K}_{m}\left(\varphi_{m}\right)-\mathcal{K}_{m}^{\prime}\left(\varphi_{m}\right)\left(Q_{n} \varphi_{m}-\varphi_{m}\right)=R\left(Q_{n} \varphi_{m}-\varphi_{m}\right) .
$$

Then by (3.16)

$$
\left\|\mathcal{K}_{m}^{\prime}\left(\varphi_{m}\right)\left(I-Q_{n}\right) y_{n}\right\|_{\infty} \leq C_{7}\left\|y_{n}\right\|_{\infty} h^{2}
$$

By $(2.8)$

$$
\left\|y_{n}\right\|_{\infty}=\left\|R\left(Q_{n} \varphi_{m}-\varphi_{m}\right)\right\|_{\infty} \leq C_{2}\left\|Q_{n} \varphi_{m}-\varphi_{m}\right\|_{\infty}^{2} .
$$

Using (2.6), (2.13) and (2.15), we obtain

$$
\left\|Q_{n} \varphi_{m}-\varphi_{m}\right\|_{\infty}=O\left(\max \left\{\tilde{h}^{2}, h^{r+1}\right\}\right) .
$$

Thus,

$$
\left\|\mathcal{K}_{m}^{\prime}\left(\varphi_{m}\right)\left(I-Q_{n}\right) y_{n}\right\|_{\infty}=O\left(h^{2} \max \left\{\tilde{h}^{2}, h^{r+1}\right\}^{2}\right) .
$$

Using (3.6) it can be checked that

$$
\left\|\mathcal{K}_{m}^{\prime}\left(\varphi_{m}\right)\left(I-Q_{n}\right) \mathcal{K}_{m}^{\prime}\left(\varphi_{m}\right)\left(I-Q_{n}\right) \varphi_{m}\right\|_{\infty}=O\left(h^{4} \max \left\{\tilde{h}^{2}, h^{r+1}\right\}\right) .
$$

The required result then follows from (3.17), (3.19) and the above estimate.

Proposition 4. Let $\varphi_{m}$ be the Nyström solution and $z_{n}^{M}$ be the discrete modified projection solution. Then

$$
\left\|\tilde{\mathcal{K}}_{n}^{M}\left(z_{n}^{M}\right)-\tilde{\mathcal{K}}_{n}^{M}\left(\varphi_{m}\right)-\left(\tilde{\mathcal{K}}_{n}^{M}\right)^{\prime}\left(\varphi_{m}\right)\left(z_{n}^{M}-\varphi_{m}\right)\right\|_{\infty}=O\left(\max \left\{\tilde{h}^{2}, h^{r+3}\right\}^{2}\right) .
$$

Proof. Note that for $m$ and $n$ big enough, $\varphi_{m}, z_{n}^{M} \in \mathcal{B}\left(\varphi, \delta_{0}\right)$. By the generalized Taylor's theorem,

$$
\begin{aligned}
\tilde{\mathcal{K}}_{n}^{M}\left(z_{n}^{M}\right) & (s)-\tilde{\mathcal{K}}_{n}^{M}\left(\varphi_{m}\right)(s)-\left(\tilde{\mathcal{K}}_{n}^{M}\right)^{\prime}\left(\varphi_{m}\right)\left(z_{n}^{M}-\varphi_{m}\right)(s) \\
= & \int_{0}^{1}(1-\theta)\left(\tilde{\mathcal{K}}_{n}^{M}\right)^{\prime \prime}\left(\varphi_{m}+\theta\left(z_{n}^{M}-\varphi_{m}\right)\right)\left(z_{n}^{M}-\varphi_{m}\right)^{2}(s) d \theta .
\end{aligned}
$$

Hence

$$
\begin{aligned}
\left\|\tilde{\mathcal{K}}_{n}^{M}\left(z_{n}^{M}\right)-\tilde{\mathcal{K}}_{n}^{M}\left(\varphi_{m}\right)-\left(\tilde{\mathcal{K}}_{n}^{M}\right)^{\prime}\left(\varphi_{m}\right)\left(z_{n}^{M}-\varphi_{m}\right)\right\|_{\infty} \\
\quad \leq \frac{1}{2} \max _{0 \leq \theta \leq 1}\left\|\left(\tilde{\mathcal{K}}_{n}^{M}\right)^{\prime \prime}\left(\varphi_{m}+\theta\left(z_{n}^{M}-\varphi_{m}\right)\right)\right\|\left\|z_{n}^{M}-\varphi_{m}\right\|_{\infty}^{2} .
\end{aligned}
$$


It can be shown that

$$
\max _{0 \leq \theta \leq 1}\left\|\left(\tilde{\mathcal{K}}_{n}^{M}\right)^{\prime \prime}\left(\varphi_{m}+\theta\left(z_{n}^{M}-\varphi_{m}\right)\right)\right\| \leq C_{8} .
$$

We skip the details. The required result then follows from Theorem 1.

Proposition 5. Let $\varphi_{m}$ be the Nyström solution and $z_{n}^{M}$ be the discrete modified projection solution. Then

$$
\left\|\mathcal{K}_{m}^{\prime}\left(\varphi_{m}\right)\left(\left(\tilde{\mathcal{K}}_{n}^{M}\right)^{\prime}\left(\varphi_{m}\right)-\mathcal{K}_{m}^{\prime}\left(\varphi_{m}\right)\right)\left(z_{n}^{M}-\varphi_{m}\right)\right\|_{\infty}=O\left(h^{2} \max \left\{\tilde{h}^{2}, h^{r+3}\right\}\right) .
$$

Proof. Note that

$$
\begin{gathered}
\mathcal{K}_{m}^{\prime}\left(\varphi_{m}\right)\left(\left(\tilde{\mathcal{K}}_{n}^{M}\right)^{\prime}\left(\varphi_{m}\right)-\mathcal{K}_{m}^{\prime}\left(\varphi_{m}\right)\right)=\mathcal{K}_{m}^{\prime}\left(\varphi_{m}\right)\left(I-Q_{n}\right)\left(\mathcal{K}_{m}^{\prime}\left(Q_{n} \varphi_{m}\right)\right. \\
\left.-\mathcal{K}_{m}^{\prime}\left(\varphi_{m}\right)\right) Q_{n}-\mathcal{K}_{m}^{\prime}\left(\varphi_{m}\right)\left(I-Q_{n}\right) \mathcal{K}_{m}^{\prime}\left(\varphi_{m}\right)\left(I-Q_{n}\right)
\end{gathered}
$$

Using (2.3) and (3.16) it can be shown that

$$
\left\|\mathcal{K}_{m}^{\prime}\left(\varphi_{m}\right)\left(I-Q_{n}\right) \mathcal{K}_{m}^{\prime}\left(\varphi_{m}\right)\right\|=O\left(h^{2}\right) .
$$

By (2.4) and (3.18),

$$
\left\|\mathcal{K}_{m}^{\prime}\left(Q_{n} \varphi_{m}\right)-\mathcal{K}_{m}^{\prime}\left(\varphi_{m}\right)\right\| \leq C_{2}\left\|Q_{n} \varphi_{m}-\varphi_{m}\right\|_{\infty}=O\left(\max \left\{\tilde{h}^{2}, h^{r+1}\right\}\right) .
$$

Since by $(2.3),\left\|\mathcal{K}_{m}^{\prime}\left(\varphi_{m}\right)\right\| \leq C_{1}$, it follows that

$$
\left\|\mathcal{K}_{m}^{\prime}\left(\varphi_{m}\right)\left(\left(\tilde{\mathcal{K}}_{n}^{M}\right)^{\prime}\left(\varphi_{m}\right)-\mathcal{K}_{m}^{\prime}\left(\varphi_{m}\right)\right)\right\|=O\left(h^{2}\right) .
$$

The required result follows using the estimate for $\left\|z_{n}^{M}-\varphi\right\|_{\infty}$ from Theorem 1 .

We now prove our main result about the order of convergence in the discrete iterated modified projection method.

Theorem 2. Let $r \geq 1, \kappa$ be of class $\mathcal{G}_{2}(r+1,0)$ and $f \in C^{r+1}[0,1]$. Let $\varphi$ be the unique solution of (1.2) and assume that 1 is not an eigenvalue of $\mathcal{K}^{\prime}(\varphi)$. Let $\mathcal{X}_{n}$ be the space of piecewise polynomials of degree $\leq r$ with respect to the partition (2.9) and $Q_{n}$ be the discrete orthogonal projection defined by (2.12). Let $\tilde{z}_{n}^{M}$ be the discrete iterated modified projection solution defined by (2.17). Then

$$
\left\|\tilde{z}_{n}^{M}-\varphi\right\|_{\infty}=O\left(\max \left\{\tilde{h}^{2}, h^{r+5}\right\}\right)
$$

Proof. We have from $(3.14)$

$$
\tilde{z}_{n}^{M}-\varphi_{m}=\mathcal{K}_{m}^{\prime}\left(\varphi_{m}\right)\left(z_{n}^{M}-\varphi_{m}\right)+O\left(\max \left\{\tilde{h}^{2}, h^{r+3}\right\}^{2}\right) .
$$


By Proposition 4.2 from Kulkarni-Rakshit [11], we have

$$
\left\|\left[I-\mathcal{K}_{m}^{\prime}\left(\varphi_{m}\right)\right]^{-1}\right\| \leq 4\left\|\left(I-\mathcal{K}^{\prime}(\varphi)\right)^{-1}\right\| .
$$

Hence by Propositions $3-5$ and the estimate (3.15),

$$
\left\|\mathcal{K}_{m}^{\prime}\left(\varphi_{m}\right)\left(z_{n}^{M}-\varphi_{m}\right)\right\|_{\infty}=O\left(\max \left\{h^{4} \max \left\{\tilde{h}^{2}, h^{r+1}\right\}, h^{2} \max \left\{\tilde{h}^{2}, h^{r+3}\right\}\right\}\right),
$$

It follows that

$$
\left\|\tilde{z}_{n}^{M}-\varphi_{m}\right\|_{\infty}=O\left(h^{2} \max \left\{\tilde{h}^{2}, h^{r+3}\right\}\right) .
$$

Since, $\tilde{z}_{n}^{M}-\varphi=\tilde{z}_{n}^{M}-\varphi_{m}+\varphi_{m}-\varphi$ and $\left\|\varphi-\varphi_{m}\right\|_{\infty}=O\left(\tilde{h}^{2}\right)$, the required result follows.

\section{Piecewise constant function approximation: $r=0$}

In this section we assume that $\kappa$ is of class $\mathcal{G}_{2}(2,0)$. If we follow the development in Section 3, then we obtain the following orders of convergence:

$$
\left\|z_{n}^{M}-\varphi\right\|_{\infty}=O\left(h^{2}\right), \quad\left\|\tilde{z}_{n}^{M}-\varphi\right\|_{\infty}=O\left(\max \left\{\tilde{h}^{2}, h^{3}\right\}\right) .
$$

But by looking at the proofs more carefully, we are able to improve the above estimates. While for $r \geq 1$, both $\left\|\mathcal{K}_{m}^{\prime}(\varphi)\left(I-Q_{n}\right) v\right\|_{\infty}$ and $\|\left(I-Q_{n}\right) \mathcal{K}_{m}^{\prime}(\varphi)(I-$ $\left.Q_{n}\right) v \|_{\infty}$ are of the same order, we could show that for $r=0$,

$$
\left\|\mathcal{K}_{m}^{\prime}(\varphi)\left(I-Q_{n}\right) v\right\|_{\infty}=O\left(h^{2}\right) \text { and }\left\|\left(I-Q_{n}\right) \mathcal{K}_{m}^{\prime}(\varphi)\left(I-Q_{n}\right) v\right\|_{\infty}=O\left(h^{3}\right) .
$$

Consider $\mathcal{X}_{n}$ to be the space of piecewise constant functions with respect to the partition (2.9). We choose Gauss 2 point rule as a basic quadrature rule:

$$
\int_{0}^{1} f(t) d t \approx w_{1} f\left(\mu_{1}\right)+w_{2} f\left(\mu_{2}\right), w_{1}=w_{2}=\frac{1}{2}, \mu_{1}=\frac{1}{2}-\frac{1}{2 \sqrt{3}}, \mu_{2}=\frac{1}{2}+\frac{1}{2 \sqrt{3}} .
$$

A composite integration rule with respect to the fine partition (2.1) is then defined as

$$
\int_{0}^{1} f(t) d t \approx \tilde{h} \sum_{i=1}^{m} \sum_{q=1}^{2} w_{q} f\left(\zeta_{q}^{i}\right), \quad \zeta_{q}^{i}=s_{i-1}+\mu_{q} \tilde{h}
$$

Recall from (2.10) that for $f, g \in C\left(\Delta_{j}\right)$,

$$
\langle f, g\rangle_{\Delta_{j}}=\tilde{h} \sum_{\nu=1}^{p} \sum_{q=1}^{2} w_{q} f\left(\zeta_{q}^{(j-1) p+\nu}\right) g\left(\zeta_{q}^{(j-1) p+\nu}\right) .
$$

The discrete orthogonal projection $Q_{n, j}: C\left(\Delta_{j}\right) \rightarrow \mathcal{P}_{0, \Delta_{j}}$ is defined as follows:

$$
\left(Q_{n, j} v\right)(t)=\frac{1}{p}\left[\sum_{\nu=1}^{p} \sum_{q=1}^{2} w_{q} v\left(\zeta_{q}^{(j-1) p+\nu}\right)\right], \quad t \in\left(t_{j-1}, t_{j}\right],
$$


and

$$
\left(Q_{n, 1} v\right)(0)=\frac{1}{p}\left[\sum_{\nu=1}^{p} \sum_{q=1}^{2} w_{q} v\left(\zeta_{q}^{\nu}\right)\right] .
$$

A discrete orthogonal projection $Q_{n}: C[0,1] \rightarrow \mathcal{X}_{n}$ is defined as

$$
Q_{n} v=\sum_{j=1}^{n} Q_{n, j} v
$$

The following result is crucial in obtaining improved orders of convergence.

Proposition 6. If $v \in C^{1}[0,1]$, then

$$
\begin{aligned}
& \left\|\left(I-Q_{n}\right) \mathcal{K}_{m}^{\prime}(\varphi)\left(I-Q_{n}\right) v\right\|_{\infty}=O\left(h^{3}\right), \\
& \left\|\mathcal{K}_{m}^{\prime}(\varphi)\left(I-Q_{n}\right) \mathcal{K}_{m}^{\prime}(\varphi)\left(I-Q_{n}\right) v\right\|_{\infty}=O\left(h^{4}\right) .
\end{aligned}
$$

Proof. Note that

$$
\left\|\left(I-Q_{n}\right) \mathcal{K}_{m}^{\prime}(\varphi)\left(I-Q_{n}\right) v\right\|_{\infty}=\max _{1 \leq i \leq n} \sup _{s \in\left[t_{i-1}, t_{i}\right]}\left|\left(I-Q_{n, i}\right) \mathcal{K}_{m}^{\prime}(\varphi)\left(I-Q_{n}\right) v(s)\right| .
$$

For $s \in\left[t_{i-1}, t_{i}\right]$,

$$
\begin{aligned}
(I & \left.-Q_{n, i}\right) \mathcal{K}_{m}^{\prime}(\varphi)\left(I-Q_{n}\right) v(s) \\
& =\frac{1}{p} \sum_{\nu=1}^{p} \sum_{q=1}^{2} w_{q}\left\{\mathcal{K}_{m}^{\prime}(\varphi)\left(I-Q_{n}\right) v(s)-\mathcal{K}_{m}^{\prime}(\varphi)\left(I-Q_{n}\right) v\left(\zeta_{q}^{(i-1) p+\nu}\right)\right\} \\
& =\frac{1}{p} \sum_{\nu=1}^{p} \sum_{q=1}^{2} \sum_{\substack{j=1 \\
j \neq i}}^{n} w_{q}\left\langle\ell_{*, s}-\ell_{*, \zeta_{q}^{(i-1) p+\nu}},\left(I-Q_{n, j}\right) v\right\rangle_{\Delta_{j}} \\
& +\frac{1}{p} \sum_{\nu=1}^{p} \sum_{q=1}^{2} w_{q}\left\langle\ell_{*, s}-\ell_{*, \zeta_{q}^{(i-1) p+\nu}},\left(I-Q_{n, i}\right) v\right\rangle_{\Delta_{i}} .
\end{aligned}
$$

For $j \neq i$,

$$
\begin{aligned}
\left\langle\ell_{*, s}-\ell_{*, \zeta_{q}^{(i-1) p+\nu}},\right. & \left.\left(I-Q_{n, j}\right) v\right\rangle_{\Delta_{j}} \\
= & \left(s-\zeta_{q}^{(i-1) p+\nu}\right)\left\langle D^{(1,0)} \ell_{*}\left(\eta_{q}^{(i-1) p+\nu}, \cdot\right),\left(I-Q_{n, j}\right) v\right\rangle_{\Delta_{j}},
\end{aligned}
$$

for some $\eta_{q}^{(i-1) p+\nu} \in\left(t_{i-1}, t_{i}\right)$. Define the following constant function

$$
g_{q}^{(i-1) p+\nu}(t)=D^{(1,0)} \ell_{*}\left(\eta_{q}^{(i-1) p+\nu}, \frac{t_{j-1}+t_{j}}{2}\right), \quad t \in\left[t_{j-1}, t_{j}\right] .
$$

Then

$$
\begin{aligned}
& \left\langle\ell_{*, s}-\ell_{*, \zeta_{q}^{(i-1) p+\nu}},\left(I-Q_{n, j}\right) v\right\rangle_{\Delta_{j}} \\
& \quad=\left(s-\zeta_{q}^{(i-1) p+\nu}\right)\left\langle D^{(1,0)} \ell_{*}\left(\eta_{q}^{(i-1) p+\nu}, \cdot\right)-g_{q}^{(i-1) p+\nu},\left(I-Q_{n, j}\right) v\right\rangle_{\Delta_{j}} .
\end{aligned}
$$


From (2.11) and (2.14),

$$
\left|\left\langle\ell_{*, s}-\ell_{*, \zeta_{q}^{(i-1) p+\nu}},\left(I-Q_{n, j}\right) v\right\rangle_{\Delta_{j}}\right| \leq C_{5}\left(\max _{s \neq t}\left|D^{(1,1)} \ell_{*}(s, t)\right|\right)\left\|v^{\prime}\right\|_{\infty} h^{4} .
$$

On the other hand, from (3.5) with $r=0$,

$$
\left|\left\langle\ell_{*, s}-\ell_{*, \zeta_{q}^{(i-1) p+\nu}},\left(I-Q_{n, i}\right) v\right\rangle_{\Delta_{i}}\right| \leq 2 C_{5} C_{6}\left\|v^{\prime}\right\|_{\infty} h^{3}, \quad \nu=1, \ldots, p .
$$

Thus, from (4.4) and the above two estimates,

$$
\begin{aligned}
& \|\left(I-Q_{n, i}\right) \mathcal{K}_{m}^{\prime}(\varphi)\left(I-Q_{n}\right) \\
& v \|_{\Delta_{i}, \infty} \\
& \leq C_{5} \max \left\{2 C_{6}, \max _{s \neq t}\left|D^{(1,1)} \ell_{*}(s, t)\right|\right\}\left\|v^{\prime}\right\|_{\infty} h^{3} .
\end{aligned}
$$

This completes the proof of (4.2). Proceeding as in the proof of Proposition 2 and using the estimate (4.2), (4.3) is proved.

Theorem 3. Let $\kappa$ be of class $\mathcal{G}_{2}(2,0)$ and $f \in C^{2}[0,1]$. Let $\varphi$ be the unique solution of (1.2) and assume that 1 is not an eigenvalue of $\mathcal{K}^{\prime}(\varphi)$. Let $\mathcal{X}_{n}$ be the space of piecewise constant functions with respect to the partition (2.9) and $Q_{n}: L^{\infty}[0,1] \rightarrow \mathcal{X}_{n}$ be the discrete orthogonal projection defined by (4.1). Let $z_{n}^{M}$ be the discrete modified projection solution in $\mathcal{B}\left(\varphi, \delta_{0}\right)$. Then

$$
\left\|z_{n}^{M}-\varphi\right\|_{\infty}=O\left(\max \left\{\tilde{h}^{2}, h^{3}\right\}\right) .
$$

Proof. Recall from (3.10) that

$$
\left\|z_{n}^{M}-\varphi\right\|_{\infty} \leq 6\left\|\left(I-\mathcal{K}^{\prime}(\varphi)\right)^{-1}\right\|\left(\left\|\mathcal{K}(\varphi)-\mathcal{K}_{m}(\varphi)\right\|_{\infty}+\left\|\mathcal{K}_{m}(\varphi)-\tilde{\mathcal{K}}_{n}^{M}(\varphi)\right\|_{\infty}\right) .
$$

From (2.5) we have

$$
\left\|\mathcal{K}(\varphi)-\mathcal{K}_{m}(\varphi)\right\|_{\infty}=O\left(\tilde{h}^{2}\right)
$$

On the other hand,

$$
\begin{aligned}
\left\|\mathcal{K}_{m}(\varphi)-\tilde{\mathcal{K}}_{n}^{M}(\varphi)\right\|_{\infty} \leq & \left\|\left(I-Q_{n}\right)\left(\mathcal{K}_{m}\left(Q_{n} \varphi\right)-\mathcal{K}_{m}(\varphi)-\mathcal{K}_{m}^{\prime}(\varphi)\left(Q_{n} \varphi-\varphi\right)\right)\right\|_{\infty} \\
& +\left\|\left(I-Q_{n}\right) \mathcal{K}_{m}^{\prime}(\varphi)\left(Q_{n} \varphi-\varphi\right)\right\|_{\infty} .
\end{aligned}
$$

Recall from (2.7) that

$$
\mathcal{K}_{m}\left(Q_{n} \varphi\right)-\mathcal{K}_{m}(\varphi)-\mathcal{K}_{m}^{\prime}(\varphi)\left(Q_{n} \varphi-\varphi\right)=R\left(Q_{n} \varphi-\varphi\right)
$$

where

$$
R\left(Q_{n} \varphi-\varphi\right)(s)=\int_{0}^{1} \mathcal{K}_{m}^{\prime \prime}\left(\varphi+\theta\left(Q_{n} \varphi-\varphi\right)\right)\left(Q_{n} \varphi-\varphi\right)^{2}(s)(1-\theta) d \theta .
$$


Let

$$
C_{9}=\max \left\{\sup _{\substack{0 \leq t<s \leq 1 \\|u| \leq\|\varphi\|_{\infty}+\delta_{0}}}\left|D^{(0,1,1)} \ell_{1}(s, t, u)\right|, \sup _{\substack{0 \leq s<t \leq 1 \\|u| \leq\|\varphi\|_{\infty}+\delta_{0}}}\left|D^{(0,1,1)} \ell_{2}(s, t, u)\right|\right\} .
$$

It can be checked that

$$
\mathcal{K}_{m}^{\prime \prime}\left(\varphi+\theta\left(Q_{n} \varphi-\varphi\right)\right)\left(Q_{n} \varphi-\varphi\right)^{2}(s)=\sum_{j=1}^{n}\left\langle\left(I-Q_{n, j}\right) \sigma_{n, s},\left(\left(I-Q_{n, j}\right) \varphi\right)^{2}\right\rangle_{\Delta_{j}},
$$

where for a fixed $s \in[0,1]$,

$$
\sigma_{n, s}(t)=\sigma_{n}(s, t)=\frac{\partial^{2} \kappa}{\partial u^{2}}\left(s, t, \varphi(t)+\theta\left(Q_{n} \varphi-\varphi\right)(t)\right), \quad t \in[0,1] .
$$

If $s=t_{i}$ for some $i$, then for all $j$ and if $s \in\left(t_{i-1}, t_{i}\right)$ for some $i$, then for $j \neq i$,

$$
\left\|\left(I-Q_{n, j}\right) \sigma_{n, s}\right\|_{\Delta_{j}, \infty} \leq C_{5} C_{9} h .
$$

We then obtain $\left\|\mathcal{K}_{m}^{\prime \prime}\left(\varphi+\theta\left(Q_{n} \varphi-\varphi\right)\right)\left(Q_{n} \varphi-\varphi\right)^{2}\right\|_{\infty}=O\left(h^{3}\right)$. It follows that

$$
\left\|\left(I-Q_{n}\right)\left(\mathcal{K}_{m}\left(Q_{n} \varphi\right)-\mathcal{K}_{m}(\varphi)-\mathcal{K}_{m}^{\prime}(\varphi)\left(Q_{n} \varphi-\varphi\right)\right)\right\|_{\infty}=O\left(h^{3}\right) .
$$

Using the estimate (4.2) of Proposition 6 and (4.7), we thus obtain

$$
\left\|\mathcal{K}_{m}(\varphi)-\tilde{\mathcal{K}}_{n}^{M}(\varphi)\right\|_{\infty}=O\left(h^{3}\right) .
$$

The required result follows from (4.6) and the above estimate.

Theorem 4. Let $\kappa$ be of class $\mathcal{G}_{2}(2,0)$ and $f \in C^{2}[0,1]$. Let $\varphi$ be the unique solution of (1.2) and assume that 1 is not an eigenvalue of $\mathcal{K}^{\prime}(\varphi)$. Let $\mathcal{X}_{n}$ be the space of piecewise constant functions with respect to the partition (2.9) and $Q_{n}: L^{\infty}[0,1] \rightarrow \mathcal{X}_{n}$ be the discrete orthogonal projection defined by (4.1). Let $\tilde{z}_{n}^{M}$ be the discrete iterated modified projection solution defined by (2.17). Then

$$
\left\|\tilde{z}_{n}^{M}-\varphi\right\|_{\infty}=O\left(\max \left\{\tilde{h}^{2}, h^{4}\right\}\right) .
$$

Proof. Recall from Section 3.3 that

$$
\tilde{z}_{n}^{M}-\varphi_{m}=\mathcal{K}_{m}^{\prime}\left(\varphi_{m}\right)\left(z_{n}^{M}-\varphi_{m}\right)+O\left(\left\|z_{n}^{M}-\varphi\right\|_{\infty}^{2}\right) .
$$

Hence by Theorem 3,

$$
\tilde{z}_{n}^{M}-\varphi_{m}=\mathcal{K}_{m}^{\prime}\left(\varphi_{m}\right)\left(z_{n}^{M}-\varphi_{m}\right)+O\left(\max \left\{\tilde{h}^{2}, h^{3}\right\}^{2}\right) .
$$

We now obtain estimates for the three terms in the expression for $\mathcal{K}_{m}^{\prime}\left(\varphi_{m}\right)\left(z_{n}^{M}-\right.$ $\left.\varphi_{m}\right)$ given in (3.15). Note that

$$
\begin{aligned}
& \left\|\mathcal{K}_{m}\left(\varphi_{m}\right)-\tilde{\mathcal{K}}_{n}^{M}\left(\varphi_{m}\right)\right\|_{\infty} \leq \|\left(I-Q_{n}\right)\left(\mathcal{K}_{m}\left(Q_{n} \varphi_{m}\right)-\mathcal{K}_{m}\left(\varphi_{m}\right)\right. \\
& \left.-\mathcal{K}_{m}^{\prime}\left(\varphi_{m}\right)\left(Q_{n} \varphi_{m}-\varphi_{m}\right)\right)\left\|_{\infty}+\right\|\left(I-Q_{n}\right) \mathcal{K}_{m}^{\prime}\left(\varphi_{m}\right)\left(Q_{n} \varphi_{m}-\varphi_{m}\right) \|_{\infty}
\end{aligned}
$$


Recall from (2.7) that

$y_{n}=\mathcal{K}_{m}\left(Q_{n} \varphi_{m}\right)-\mathcal{K}_{m}\left(\varphi_{m}\right)-\mathcal{K}_{m}^{\prime}\left(\varphi_{m}\right)\left(Q_{n} \varphi_{m}-\varphi_{m}\right)=R\left(Q_{n} \varphi_{m}-\varphi_{m}\right)$

Now proceeding as in the proof of Theorem 3, we obtain

$$
\left\|y_{n}\right\|_{\infty}=\left\|R\left(Q_{n} \varphi_{m}-\varphi_{m}\right)\right\|_{\infty}=O\left(\max \left\{\tilde{h}^{2}, h^{3}\right\}\right)
$$

Note that

$$
\left\|\mathcal{K}_{m}^{\prime}\left(\varphi_{m}\right)\left(I-Q_{n}\right) y_{n}\right\|_{\infty} \leq C_{7}\left\|y_{n}\right\|_{\infty} h=O\left(h \max \left\{\tilde{h}^{2}, h^{3}\right\}\right)
$$

Using (4.3) it can be seen that

$$
\left\|\mathcal{K}_{m}^{\prime}\left(\varphi_{m}\right)\left(I-Q_{n}\right) \mathcal{K}_{m}^{\prime}\left(\varphi_{m}\right)\left(I-Q_{n}\right) \varphi_{m}\right\|_{\infty}=O\left(h^{4}\right)
$$

Thus, from (4.11), (4.12) and the above estimate, we obtain

$$
\left\|\mathcal{K}_{m}^{\prime}\left(\varphi_{m}\right)\left(\mathcal{K}_{m}\left(\varphi_{m}\right)-\tilde{\mathcal{K}}_{n}^{M}\left(\varphi_{m}\right)\right)\right\|_{\infty}=O\left(h \max \left\{\tilde{h}^{2}, h^{3}\right\}\right) .
$$

We recall the following result from Proposition 4:

$$
\begin{aligned}
\| \tilde{\mathcal{K}}_{n}^{M}\left(z_{n}^{M}\right)-\tilde{\mathcal{K}}_{n}^{M}\left(\varphi_{m}\right)-\left(\tilde{\mathcal{K}}_{n}^{M}\right)^{\prime}\left(\varphi_{m}\right)\left(z_{n}^{M}-\varphi_{m}\right) & \\
& \leq C_{8}\left\|z_{n}^{M}-\varphi_{m}\right\|_{\infty}^{2}=O\left(\max \left\{\tilde{h}^{2}, h^{3}\right\}^{2}\right) .
\end{aligned}
$$

Note that

$$
\left\|\mathcal{K}_{m}^{\prime}\left(\varphi_{m}\right)\left(\left(\tilde{\mathcal{K}}_{n}^{M}\right)^{\prime}\left(\varphi_{m}\right)-\mathcal{K}_{m}^{\prime}\left(\varphi_{m}\right)\right)\right\|=O(h)
$$

Hence

$$
\left\|\mathcal{K}_{m}^{\prime}\left(\varphi_{m}\right)\left(\left(\tilde{\mathcal{K}}_{n}^{M}\right)^{\prime}\left(\varphi_{m}\right)-\mathcal{K}_{m}^{\prime}\left(\varphi_{m}\right)\right)\left(z_{n}^{M}-\varphi_{m}\right)\right\|_{\infty}=O\left(h \max \left\{\tilde{h}^{2}, h^{3}\right\}\right) .
$$

We thus obtain the following estimate using (3.15), (4.13)-(4.15):

$$
\left\|\mathcal{K}_{m}^{\prime}\left(\varphi_{m}\right)\left(z_{n}^{M}-\varphi_{m}\right)\right\|_{\infty}=O\left(h \max \left\{\tilde{h}^{2}, h^{3}\right\}\right) .
$$

From (4.10) it follows that $\left\|\tilde{z}_{n}^{M}-\varphi_{m}\right\|_{\infty}=O\left(h \max \left\{\tilde{h}^{2}, h^{3}\right\}\right)$. Since $\left\|\varphi-\varphi_{m}\right\|_{\infty}=$ $O\left(\tilde{h}^{2}\right)$, the required result follows.

Remark 2. It can be shown that

$$
\left\|z_{n}^{G}-\varphi\right\|_{\infty}=O(h), \quad\left\|z_{n}^{S}-\varphi\right\|_{\infty}=O\left(h^{2}\right)
$$




\section{$5 \quad$ Numerical results}

For the sake of illustration, we quote some numerical results from Grammont et al [7] for the following example considered in Atkinson-Potra [3].

Consider

$$
x(s)-\int_{0}^{1} \kappa(s, t)\left[f(t, x(t)] d t=\int_{0}^{1} \kappa(s, t) z(t) d t, \quad 0 \leq s \leq 1,\right.
$$

where

$$
\kappa(s, t)=\left\{\begin{array}{ll}
(1-s) t, & 0 \leq t \leq s \leq 1, \\
s(1-t), & 0 \leq s \leq t \leq 1,
\end{array} \quad \text { and } \quad f(t, u)=\frac{1}{1+t+u}\right.
$$

with $z(t)$ so chosen that $\varphi(t)=\frac{t(1-t)}{t+1}$ is the solution of (5.1). In this example, $r$ can be chosen as large as we want.

\subsection{Piecewise constant functions $(r=0)$}

Let $\mathcal{X}_{n}$ be the space of piecewise constant functions with respect to the partition (2.9) and $Q_{n}: L^{\infty}[0,1] \rightarrow \mathcal{X}_{n}$ be the discrete orthogonal projection defined by (4.1). The numerical quadrature is chosen to be the composite Gauss 2 rule with respect to partition (2.1) with $m=n^{2}$ subintervals. Then $\tilde{h}=h^{2}$.

In Table 1 and Table 2, the computed orders of convergence in the discrete Galerkin, discrete iterated Galerkin, discrete Modified Projection and the discrete iterated Modified Projection methods are denoted respectively by $\delta_{G}, \delta_{S}, \delta_{M}$ and $\delta_{I M}$. It can be seen from Table 1 that the computed values of order of convergence match well with the theoretically predicted values in (4.5), (4.9) and (4.16).

Table 1. Piecewise constant functions

\begin{tabular}{ccccccccc}
\hline$n$ & $\left\|\varphi-z_{n}^{G}\right\|_{\infty}$ & $\delta_{G}$ & $\left\|\varphi-z_{n}^{S}\right\|$ & $\delta_{S}$ & $\left\|\varphi-z_{n}^{M}\right\|_{\infty}$ & $\delta_{M}$ & $\left\|\varphi-\tilde{z}_{n}^{M}\right\|_{\infty}$ & $\delta_{I M}$ \\
\hline 2 & $1.22 e-1$ & & $8.40 e-3$ & & $4.34 \times 10^{-3}$ & & $5.23 \times 10^{-3}$ & \\
4 & $8.65 e-2$ & 0.49 & $2.35 e-3$ & 1.84 & $4.31 \times 10^{-4}$ & 3.33 & $3.14 \times 10^{-4}$ & 4.06 \\
8 & $5.09 e-2$ & 0.77 & $6.22 e-4$ & 1.92 & $5.28 \times 10^{-5}$ & 3.03 & $1.89 \times 10^{-5}$ & 4.05 \\
16 & $2.70 e-2$ & 0.91 & $1.59 e-4$ & 1.96 & $6.92 \times 10^{-6}$ & 2.93 & $1.36 \times 10^{-6}$ & 3.80 \\
32 & $1.33 e-2$ & 1.02 & $4.02 e-5$ & 1.98 & $8.38 \times 10^{-7}$ & 3.05 & $4.55 \times 10^{-8}$ & 4.90 \\
\hline
\end{tabular}

\subsection{Piecewise linear functions $(r=1)$}

Let $\mathcal{X}_{n}$ be the space of piecewise linear polynomials w.r.t. the partition (2.9) and $Q_{n}$ be the discrete orthogonal projection defined by (2.12). We choose the composite Gauss 2 point rule with $n^{2}$ intervals for the Galerkin methods and the composite Gauss 2 point rule with $n^{3}$ intervals for the modified projection methods. In the latter case $\tilde{h}^{2}=h^{6}$. 
Table 2. Piecewise linear functions

\begin{tabular}{ccccccccc}
\hline$n$ & $\left\|\varphi-\varphi_{n}^{G}\right\|_{\infty}$ & $\delta_{G}$ & $\left\|\varphi-\varphi_{n}^{S}\right\|$ & $\delta_{S}$ & $\left\|\varphi-\varphi_{n}^{M}\right\|_{\infty}$ & $\delta_{M}$ & $\left\|\varphi-\tilde{\varphi}_{n}^{M}\right\|_{\infty}$ & $\delta_{I M}$ \\
\hline 2 & $1.32 e-1$ & & $4.97 e-3$ & & $1.54 \times 10^{-3}$ & & $1.34 \times 10^{-3}$ & \\
4 & $4.98 e-2$ & 1.41 & $4.46 e-4$ & 3.48 & $1.12 \times 10^{-4}$ & 3.78 & $1.89 \times 10^{-5}$ & 6.15 \\
8 & $1.58 e-2$ & 1.66 & $3.89 e-5$ & 3.52 & $1.06 \times 10^{-5}$ & 3.40 & $2.48 \times 10^{-7}$ & 6.25 \\
16 & $4.51 e-3$ & 1.81 & $3.15 e-6$ & 3.62 & $9.10 \times 10^{-7}$ & 3.54 & $2.92 \times 10^{-9}$ & 6.41 \\
\hline
\end{tabular}

It can be seen from Table 2 that the computed values of the orders of convergence match well with the theoretically predicted values in (3.11), (3.13) and (3.20).

\section{References}

[1] K.E. Atkinson. The numerical evaluation of fixed points for completely continuous operators. SIAM Journal on Numerical Analysis, 10(5):799-807, 1973. https://doi.org/10.1137/0710065.

[2] K.E. Atkinson, I. Graham and I. Sloan. Piecewise continuous collocation for integral equations. SIAM Journal on Numerical Analysis, 20(1):172-186, 1983. https://doi.org/10.1137/0720012.

[3] K.E. Atkinson and F.A. Potra. Projection and iterated projection methods for nonlinear integral equations. SIAM Journal on Numerical Analysis, 24(6):13521373, 1987. https://doi.org/10.1137/0724087.

[4] K.E. Atkinson and F.A. Potra. The discrete Galerkin method for nonlinear integral equations. Journal of Integral Equations and Applications, 1(1):17-54, 1988. https://doi.org/10.1216/JIE-1988-1-1-17.

[5] L. Grammont. A Galerkin's perturbation type method to approximate a fixed point of a compact operator. International Journal of Pure and Applied Mathematics, 69(1):1-14, 2011.

[6] L. Grammont and R.P. Kulkarni. A superconvergent projection method for nonlinear compact operator equations. C.R. Acad. Sci. Paris, 342(3):215-218, 2006. https://doi.org/10.1016/j.crma.2005.11.011.

[7] L. Grammont, R.P. Kulkarni and T.J. Nidhin. Modified projection method for Urysohn integral equations with non-smooth kernels. Journal of Computational and Applied Mathematics, 294:309-322, 2016. https://doi.org/10.1016/j.cam.2015.08.020.

[8] M.A. Krasnosel'skii. Topological methods in the theory of nonlinear integral equations. Macmillan, New York, 1964.

[9] M.A. Krasnosel'skii, G.M. Vainikko, P.P. Zabreiko, Y.B. Ruticki and V.V. Stet'senko. Approximate solution of operator equations. P. Noordhoff, Groningen, 1972.

[10] M.A. Krasnosel'skii and P.P. Zabreiko. Geometrical methods of nonlinear analysis. Springer-Verlag, Berlin, 1984.

[11] R.P. Kulkarni and G. Rakshit. Discrete modified projection method for Urysohn integral equations with smooth kernels. Applied Numerical Mathematics, 126:180-198, 2018. https://doi.org/10.1016/j.apnum.2017.12.008. 